Check for updates

Cite this: RSC Adv., 2017, 7, 29464

Received 20th March 2017

Accepted 1st June 2017

DOI: $10.1039 / \mathrm{c} 7 \mathrm{ra03259e}$

rsc.li/rsc-advances

\title{
Low voltage driven surface micro-flow by Joule heating $\dagger$
}

\author{
H. Wang, ${ }^{a}$ S. G. Wang, ${ }^{a}$ J. J. Kan, ${ }^{a}$ X. Y. Deng, ${ }^{a}$ W. C. Wang, (D *a M. H. Wu ${ }^{\star b}$ \\ and Y. Lei $(\mathbb{D} *$ ac
}

We report an electrically controlled micro-flow of liquid on Au-patterned glass surfaces with a driving voltage lower than 10 volts. The Joule heating produced by electrical current passing through the $\mathrm{Au}$ layer activates the selective wetting of liquids, performing complicated micro-flow control over predesigned circuits. Transportation and serial dilution of the dye molecules in the liquid are further demonstrated.

Manipulation of small amounts of liquid is of high interest in different research fields of biology, physics and chemistry, which has triggered extensive investigations into microfluidics. ${ }^{1-3}$ Basically, microscopic liquid flow (droplet) control is one of the essential parts of microfluidics to perform complex chemical and biochemical reactions as well as analytical tasks. Typically, microfluidics is done inside closed channels to guide fluids/droplets owing to easy integration of pumps and valves for control. ${ }^{4,5}$ The integration of pumps and valves often requires size orders of magnitude which are greater than those of the microfluidic channels themselves. ${ }^{6}$ Alternatively, electrical fields and lights have been proposed and demonstrated for the actuations of fluids. However, these processes usually suffer from complicated electrode design, high voltage required to create electro-osmotic effect, poor actuations, and limiting of specialized materials. ${ }^{7-12}$

Typically, the fabrication of closed channel microfluidic chips often requires a multi-step microfabrication process. ${ }^{13}$ To simplifying the process, open microfluidics has attracted increasing attention, which is consisted of open channels or hydrophobic/hydrophilic patterns on the substrate surfaces. Moreover, the open microfluidics bears intrinsic advantages of real time sensitivity to environments and straightforward data acquisition. ${ }^{14,15}$ Owing to difficulties to integrate valves and pumps on open microfluidics, capillary force, temperature gradient and Laplace pressure caused by drop size have been utilized to actuate the liquids. ${ }^{16-19}$ However, functions like

anstitute of Nanochemistry and Nanobiology, School of Environmental and Chemical Engineering, Shanghai University, 99 Shangda Road, Shanghai, 200444, P. R. China. E-mail: wenchong@shu.edu.cn

${ }^{b}$ Shanghai Applied Radiation Institute, Shanghai University, 200444, P. R. China. E-mail: mhwu@staff.shu.edu.cn

'Institut für Physik, IMN MacroNano (ZIK), Technische Universität Ilmenau, Ilmenau, 98693, Germany.E-mail: yong.lei@tu-ilmenau.de

$\dagger$ Electronic supplementary information (ESI) available. See DOI: $10.1039 / \mathrm{c} 7 \mathrm{ra} 03259 \mathrm{e}$ valves that are fully perform complex demanding like actuation on demand are still less explored. Similarly as in the closed channels, electrical field is applied to manipulate the liquid droplets with complicated circuits and high voltages up to thousands volts. ${ }^{20-22}$ Here in this letter, we report an innovative way to control the micro-flow simply by joule heating. The liquid is confined on a thin metal layer on OTS modified glasses, controlled to wetting on desired channels upon applying voltage less than 10 volts. Mixing of the flow upon demanding is further demonstrated.

Selectively wetting/absorption of liquid on hydrophilic/ hydrophobic patterned surfaces have been extensively explored both theoretically and experimentally. ${ }^{23,24}$ For examples, water can be confined on the hydrophilic areas owing to surface tension, and be controlled to uni-directionally wet the surfaces. The fluids spread over all the possible channels due to identical surface chemistry, as schematically shown in Fig. 1a and $\mathrm{b}$. When a liquid drop is put on a hydrophilic/hydrophobic structured surface, it wets the channels owing to the contact angle difference (Fig. 1a). ${ }^{25}$ The liquid propagates along the $\mathrm{Au}$ and split into 3 flow fronts when it comes to the crossing.

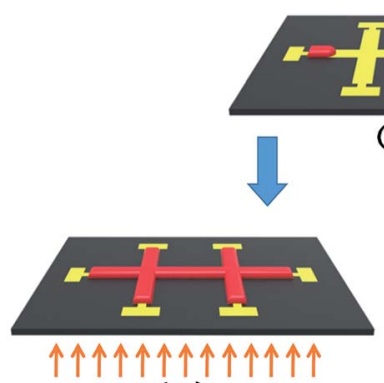

(b)

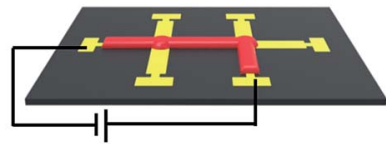

(c)
Fig. 1 Schematic representation of liquid propagation on $\mathrm{Au}$ patterned surfaces. (a) Liquid wetting on a single channel, (b) propagation upon uniformly heating, (c) propagation upon selective heating. 
Finally, the liquid will cover all the Au surface area, as shown in Fig. 1b. The spreading of the liquid over channels involves diffusion of molecules which is thermally activated. ${ }^{26}$ That is to say, flow control on desired channel can be achieved when it is selectively heated up. The selective heating of the channel is to activate molecule diffusion over specific area, followed by the fluid flow control as schematically shown in Fig. 1c.

To prove the concept experimentally, we simply used molten candle wax as the liquid, OTS modified glass as the substrates and Au stripes as the channels. The wax was chosen for their stable chemistry and low vapor pressure in air. The contact angle of molten wax on OTS modified glass and Au covered glass are $95^{\circ}$ and $75^{\circ}$ respectively (see ESI S1 $\dagger$ ). The OTS modified glass substrates were processed by standard photoresist spin coating, baking, UV exposure through a mask and development. After the pattern formation on photoresist, the samples were coated with a $3 \mathrm{~nm} \mathrm{Cr}$ adhesion layer and $20 \mathrm{~nm}$ Au layer by thermal evaporation, followed by lift off to transfer the patterns onto glass surfaces. The Au stripes are consisted of 4 groups with width of 750, 550, 450 and $400 \mu \mathrm{m}$ respectively, as shown in the left of the Fig. 2a. Each group connects to a rectangle Au pad to hold the liquid and includes multiple lines for reproducibility of the wetting process. Firstly we investigated the wetting dynamics of the candle wax on $\mathrm{Au}$ stripes patterned OTS/glass. The samples were prepared by putting the wax on Au pads. Upon the heating the sample up to $80{ }^{\circ} \mathrm{C}$ by a hot plate, the wax melts into liquid and propagates along $\mathrm{Au}$ stripes due to complete wetting property, forming wax liquid stripes on Au as shown in Fig. 2a of an optical microscope image. Obviously from the image, the wetting velocity on $\mathrm{Au}$ is stripe width dependent, i.e., the velocity
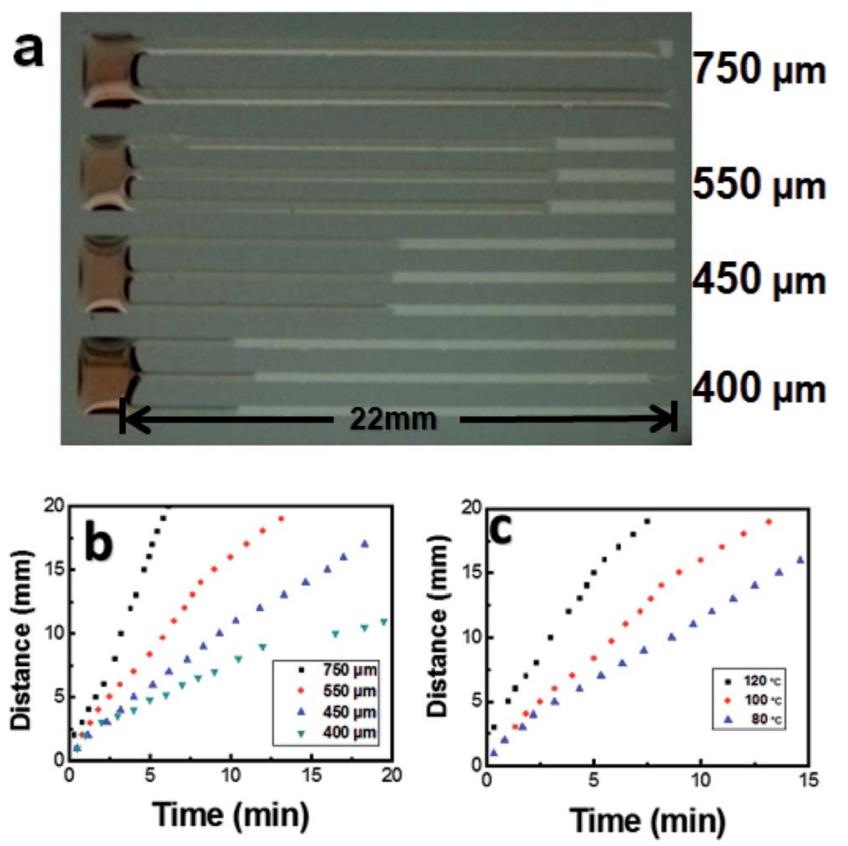

Fig. 2 (a) Photograph of candle wax wetting on Au patterned surfaces at $80^{\circ} \mathrm{C}$, (b and $\mathrm{c}$ ) progress of the wax liquid meniscus with time: (b) with width and (c) with temperature. increases the width. We note that there are almost no propagation of wax liquid observed when the stripe width is below $200 \mu \mathrm{m}$ (see ESI S2 $\dagger$ ). The wax liquid front will stop as it covers the Au stripe completely, as shown in top Au stripes in width of $750 \mu \mathrm{m}$ in the Fig. 2a.

Fig. $2 \mathrm{~b}$ shows the temporal evolution of liquid wax wetting on $\mathrm{Au}$ by plotting wetting distance over time with different stripe width. For all the Au stripes width, the propagation distance shows a saturation tendency with time. Studies on wetting dynamics show a complicated process where the capillary and precursor diffusion play crucial roles. ${ }^{27,28}$ Typically the wetting distance $L$ increases with time as a power law $L=D t^{\chi}$, where $D$ is the prefactor and $t$ is the time. According to different material systems used, $\chi$ ranging from $1 / 10$ to $1 / 2$ is theoretically predicted and experimentally observed. ${ }^{29,30}$ The impact of temperature on wetting process was also investigated and shown in Fig. 2c, where the fluid on $550 \mu \mathrm{m} \mathrm{Au}$ stripes at substrate temperature of $80^{\circ} \mathrm{C}, 100^{\circ} \mathrm{C}$ and $120^{\circ} \mathrm{C}$ are presented. The wetting velocity increases with the temperature. Contact angle measurements show that almost no wettability change of wax on Au covered glass at the $80^{\circ} \mathrm{C}$, $100{ }^{\circ} \mathrm{C}$ and $120^{\circ} \mathrm{C}$. The contact angle keeps at about $75^{\circ}$ (see ESI S3 $\dagger^{\dagger}$ ). In the case, the dynamics is thermal activated which the diffusion of molecules plays a crucial role. Besides the width and temperature, we believe the wettability of the stripe could also be influence by many factors such as surface chemistry and microscopic structure. ${ }^{31}$ In the cases, the wetting dynamics can further be tuned with surface modification and structures.

It is well known that electrical current passing through a conducting material can produce Joule heat. In some cases, the Joule heat is undesirable when electrical field was used to pump the liquid, which can cause current flow through the liquid and lead to significant increasing of the temperature. ${ }^{32}$ In the other cases, the Joule heating was also used for control of the microfluidic chip temperature by simply fabrication of thin metal layer adjacent to the fluidic channels. ${ }^{33}$ Inspired by the temperature activation of the molecule diffusion on surface, we can further control the liquid flow over the desired channels. Crossed Au stripes on OTS/glass were designed for demonstration, as schematically shown in Fig. 3a with the details of the specific size. The stripes are $550 \mu \mathrm{m}$ in width, which are attached by an electrical pad (labelled as 1 to 6 in Fig. 3a) each for the power source connection. The stripes and electrical pads are connected with $\mathrm{Au}$ dashes with width of $20 \mu \mathrm{m}$ to keep the liquid overflow to the pads. A small pad is added near the pad 1 to accommodate the wax liquid.

With a $20 \mathrm{~nm}$ thick Au layer deposited by vacuum deposition, we measured the electrical resistivity of the Au stripes by applying a $1 \mathrm{~V}$ voltage between pad 1 to pad 2-6 respectively, shown in Fig. $3 \mathrm{~b}$. The $I-V$ curves show geometry symmetry of the pattern, with electrical resistance of about $140 \Omega$ for pads 2 and $6,180 \Omega$ for pads 3 and 5 , and $200 \Omega$ for pad 4, respectively. By applying voltages between the selected electrodes, electrical current can be generated simply based on the Ohm's law. The electrical current further induces Joule heating which results in heating up of the selected Au wire. According to the power 


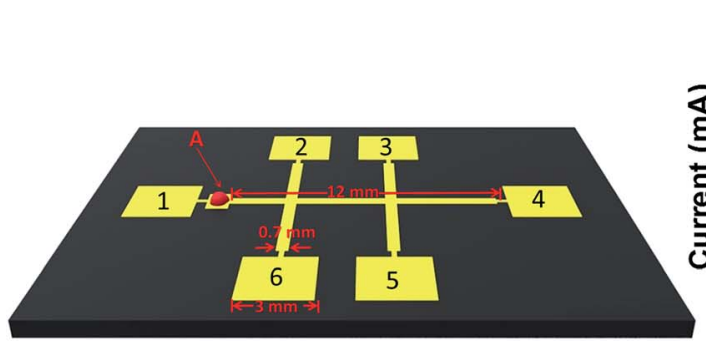

(a)

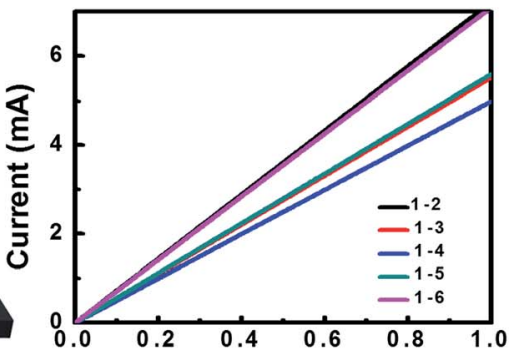

(b) Voltage (v)

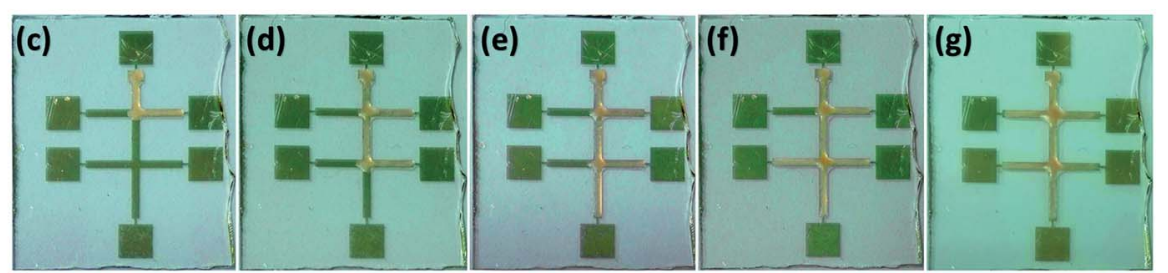

Fig. 3 (a) Schematic view of the electrical controlled surface micro-flow control, (b) I-V curves of the pad 1 to pad 2-6, respectively, and (c-g) selective wetting of the molten wax by applying voltage between pad 1 and (c) pad 2, (d) pad 3, (e) pad 4, (f) pad 5 and (g) pad 6 .

dissipated law of $P=I^{2} R$, the heating power is directly determined by the electrical current when the resistance $R$ is fixed. Fig. $3 \mathrm{c}-\mathrm{g}$ show the micro-flow control of the candle wax by applying voltage between pad 1 and pad 2 to 6 successively. The solid wax was first put on the small pad adjacent to the pad 1. As the voltage between pad 1 and pad 2 was increased to 8 volt, the wax started to melt and be held on the small pad. Further increasing the voltage to 10 volt which the temperature was estimated to be about $80^{\circ} \mathrm{C}$, the molten wax began to flow on the Au stripe. By fixing the voltage at 10 volt, the flow propagated along the Au wires and reached the first crossing, and turned the direction toward pad 2 due to the joule heating of the underneath Au stripe. Finally the flow stopped at the end of the $700 \mu \mathrm{m} \mathrm{Au}$ stripe. Using the same sample, we further applied the voltage to pad 1 and pad 3 to 6 successively, and controlled the micro-flow on the corresponding stripes, as shown in Fig. 3d-g. During the experiments, the flow stopped immediately as the applied voltage withdrew. As a comparison, we performed the experiments on a hot plate heated samples, the liquid flows simply over all the stripes (see ESI S4†). In addition, we note that the method is not limited to the wax, rather can be extended to many other materials. For examples, $N$-ethyl-D-glucamine (NEDG) and 2-hydroxyethylpyridine can be selectively driven to wet the desired channels by simply add voltage to the corresponding electrical pads (see ESI S5†).

As the result, by applying voltage between arbitrary electrodes, we can control the micro-flow on the corresponding channels, as shown in Fig. 4. Using pad 1 as the common electrode, the micro-flow can be directed to pad 6 (Fig. 4 a) or pad 2 (Fig. $4 \mathrm{~b}$ ) by selecting the corresponding pads. The proposed technique here can also be used to transport functional materials. For demonstration, we choose $N, N^{\prime}$-bis(1-naphthyl)- $N, N^{\prime}$ diphenyl-1,1'-biphenyl-4,4'-diamine (NPB, a blue dye molecule widely used for color labelling and organic electronics). ${ }^{34}$ The NPB molecule was mixed to the candle wax with weight ratio of
$1: 1000$. The fluorescence microscope images of the bottom of Fig. $4 \mathrm{a}$ and $\mathrm{b}$ show a strong blue color from the corresponding channels, confirming the successful transport the dye molecule with the flow.

Serial dilution is one of the most fundamental widely practiced microfluidic techniques, with applications ranging from measuring detector response to determining kinetic rate constants. ${ }^{35}$ We further demonstrate the ability to mix and dilute the liquid that analog to the closed channels in serial, as shown in Fig. 5. In the experiments, we fabricated a branched design with the same Au wire in width of $700 \mu \mathrm{m}$, and the electrode pads labelled as 1-4. Firstly, the NPB doped wax was used to selectively wet the top channel between pad 1 and 4 ; then the as (a)

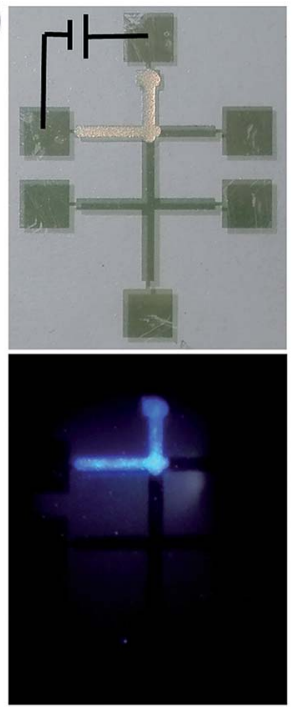

(b)

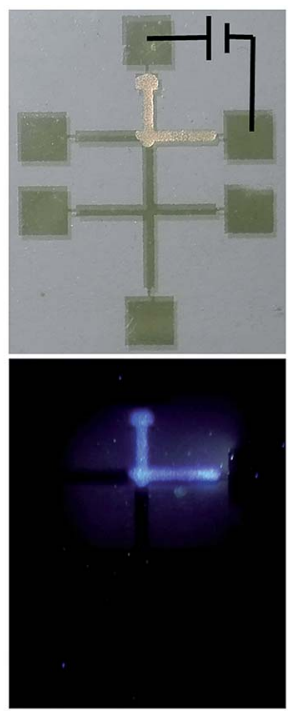

Fig. 4 Photography of wetting NPB doped wax wetting control on channels by applying voltage between pad 1 and (a) pad 6 and (b) pad 2, bottom: fluorescence photography. 


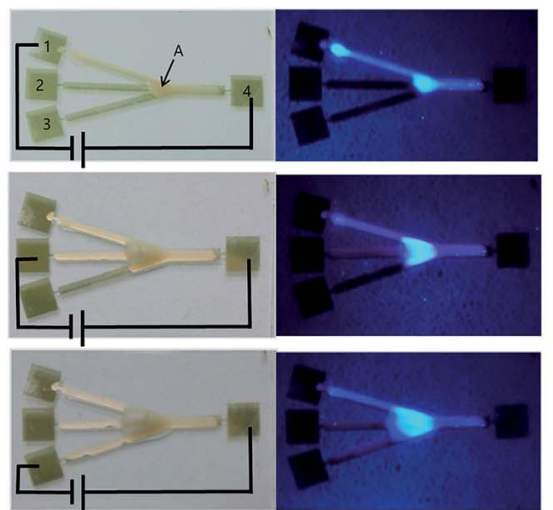

(a)

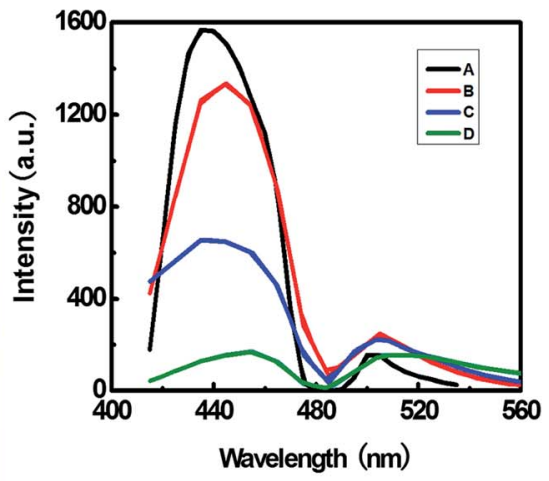

(b)

Fig. 5 (a) Photography of serial diluting of NPB doped wax, under illumination of white light (left) and UV light (right), and (b) PL spectra taken at position A marked in (a).

purchased was used for middle and low channel to dilute the NPB concentration in the junction of the branched structure as shown in Fig. 5a. We carried out the photoluminescence (PL) measurement to identify dilution effect, at the position which the three branched channels meet and marked as $\mathrm{A}$ in the Fig. 5a. First of all, the as-purchased candle wax is controlled to flow from pad 1 to 4 to be utilized as a reference sample of the NPB-doped ones. The PL spectroscopic measurement of aspurchased candle wax (Fig. 5b, curve D) shows two broad features peaked at $450 \mathrm{~nm}$ and $510 \mathrm{~nm}$ which can be attributed to the impurities. ${ }^{36}$ When the NPB doped candle wax is controlled to wet the channel between pad 1 and pad 4, the fluorescence microscope image shows strong blue color over the channel (Fig. 5a, left). The PL spectroscope shows that the feature peaked at $510 \mathrm{~nm}$ is kept almost unchanged (Fig. 5b, curve A), indicating that the amount of candle wax can be controlled by the template dimensions. However, the intensity of the feature peaked at $450 \mathrm{~nm}$ increases from the reference of 200 to 1600 , confirming the presence of the NPB molecules. To dilute the NPB dye molecules, we used as purchased wax on channels 2 and channel 3. When the as purchased wax reaches at junction position of the branched structure, the NPB molecules can be diluted owing increasing of wax amount. The intensity of the feature peaked at $450 \mathrm{~nm}$ can further be decreased to 1300 and 700 by switching the applied voltage from pad 2 to pad 3 and pad 4 successively (Fig. 5b, curve B and curve C). The decrease of NPB emission enables us to serial mix and dilution of the dye molecules upon electrical control. In principal, the electrical control surface microfluidic technique can be extended to other materials and structures of interests.

In conclusion, we have demonstrated a feasible, low voltage control of surface micro-flow simply by Joule heating on a thin metal layer. The temperature controlled by the Joule heating enables diffusion and wetting of molecules on selected channels. We further show the versatility of the technique with transporting of the functional materials and serial diluting of the liquid upon required. We believe that the technique is not limited to wax and the control can even be programed owing the compatibility to lithography and low driven voltage.

\section{Acknowledgements}

This work was financially supported by The Program for Special Appointment (Eastern Scholar) at Shanghai Institute of Higher Learning, National Natural Science Foundation of China (21577086), Shanghai Thousand Talent Plan, European Research Council (ThreeDsurface, 240144), European Research Council (HiNaPc, 737616), German Research Foundation (DFG: LE 2249_4-1).

\section{References}

1 D. G. Rackus, M. H. Shamsiab and A. R. Wheeler, Electrochemistry, Biosensors and Microfluidics: a Convergence of Fields, Chem. Soc. Rev., 2015, 44, 53205340 .

2 T. M. Squires and S. R. Quake, Microfluidics: Fluid Physics at the Nanoliter Scale, Rev. Mod. Phys., 2005, 77, 977-1026.

3 M. J. Sun, G. Q. Song, J. Q. Liu, H. M. Chen and F. Q. Nie, In situ controllable synthesis of cotton-like polyaniline nanostructures for a $\mathrm{H}_{2} \mathrm{O}_{2}$ sensor using an embedded three-electrode microfluidic chip, RSC Adv., 2017, 7, 1363713642.

4 L. Shui, R. A. Hayes, M. Jin, X. Zhang, P. Bai, A. Van den Berg and G. Zhou, Microfluidics for Electronic Paper-like Displays, Lab Chip, 2014, 14, 2374-2384.

5 L. Gervais, N. De Rooij and E. Delamarche, Microfluidic Chips for Point-of-care Immunodiagnostics, Adv. Mater., 2011, 23, 151-176.

6 C. K. Byun, K. Abisamra, Y. K. Cho and S. Takayama, Pumps for Microfluidic Cell Culture, Electrophoresis, 2014, 35, 245257.

7 A. Bandopadhyay, D. Tripathi and S. Chakraborty, Electroosmosis-modulated Peristaltic Transport in Microfluidic Channels, Phys. Fluids, 2016, 28, 052002.

8 N. Mavrogiannis, M. Desmond and Z. R. Gagnon, Fluidic Dielectrophoresis: the Polarization and Displacement of Electrical Liquid Interfaces, Electrophoresis, 2015, 36, 13861395. 
9 D. Baigl, Photo-actuation of Liquids for Light-driven Microfluidics: State of the Art and Perspectives, Lab Chip, 2012, 12, 3637-3653.

10 C. Monat, P. Domachuk, C. Grillet, M. Collins, B. J. Eggleton, M. C. Golomb, S. Mutzenich, T. Mahmud, G. Rosengarten and A. Mitchell, Optofluidics: a Novel Generation of Reconfigurable and Adaptive Compact Architectures, Microfluid. Nanofluid., 2008, 4, 81-95.

11 J. Delville, M. R. S. Vincent, R. D. Chroll, S. H. Chraïbi, B. Issenmann, R. Wunenburger, D. Lasseux, W. W. Zhang and E. Brasselet, Laser Microfluidics: Fluid Actuation by Light, J. Opt. A: Pure Appl. Opt., 2009, 11, 034015.

12 J. A. Lv, Y. Y. Liu, J. Wei, E. Q. Chen, L. Qin and Y. L. Yu, Photocontrol of Fluid Slugs in Liquid Crystal Polymer Microactuators, Nature, 2016, 537, 179-184.

13 K. H. Ren, J. H. Zhou and H. K. Wu, Materials for Microfluidic Chip Fabrication, Acc. Chem. Res., 2013, 46, 2396-2406.

14 P. Calcagnile, T. Dattoma, E. Scarpa, A. Qualtieri, L. Blasi, M. De Vittorio and F. Rizzi, A 2D approach to surfacetension-confined fluidics on parylene C, RSC Adv., 2017, 7, 15964-15970.

15 H. Wang, W. C. Wang, L. Q. Li, J. Zhu, W. X. Wang, D. Q. Zhang, Z. X. Xie, H. Fuchs, Y. Lei and L. F. Chi, Surface Microfluidic Patterning and Transporting Organic Small Molecules, Small, 2014, 13, 2549-2552.

16 E. K. Sackmann, A. L. Fulton and D. J. Beebe, The Present and Future Role of Microfluidics in Biomedical Research, Nature, 2014, 507, 181-189.

17 N. T. Nguyen, W. W. Pang and X. Huang, Sample Transport with Thermocapillary Force for Microfluidics, J. Phys.: Conf. Ser., 2006, 34, 967-972.

18 J. G. Shackman, M. S. Munson and D. Ross, Temperature Gradient Focusing for Microchannel Separations, Anal. Bioanal. Chem., 2007, 387, 155-158.

19 S. Barkley, S. J. Scarfe, E. R. Weeksb and K. Dalnoki-Veress, Predicting the Size of Droplets Produced through Laplace Pressure Induced Snap-off, Soft Matter, 2016, 12, 7398-7404.

20 X. Li, P. Zwanenburga and X. Y. Liu, Magnetic Timing Valves for Fluid Control in Paper-based Microfluidics, Lab Chip, 2013, 13, 2609-2714.

21 M. Abdelgawad and A. R. Wheeler, The Digital Revolution: A New Paradigm for Microfluidics, Adv. Mater., 2009, 21, 920925.

22 T. Um, J. Hong, D. J. Im, S. J. Lee and I. S. Kang, Electrically Controllable Microparticle Synthesis and Digital Microfluidic Manipulation by Electric-Field-Induced
Droplet Dispensing into Immiscible Fluids, Sci. Rep., 2016, 6, 31901.

23 S. Wang, T. Q. Wang, P. Ge, P. H. Xue, S. S. Ye, H. X. Chen, Z. B. Li, J. H. Zhang and B. Yang, Controlling Flow Behavior of Water in Microfluidics with a Chemically Patterned Anisotropic Wetting Surface, Langmuir, 2015, 31, 4032-4039.

24 S. Y. Xing, J. Jiang and T. Pan, Interfacial Microfluidic Transport on Micropatterned Superhydrophobic Textile, Lab Chip, 2013, 13, 1937-1947.

25 T. Guo, T. Meng, W. Li, J. Qin, Z. Tong, Q. Zhang and X. Li, UV-driven Microvalve Based on a Micro-nano $\mathrm{TiO}_{2} / \mathrm{SiO}_{2}$ Composite Surface for Microscale Flow Control, Nanotechnology, 2014, 25, 125301.

26 R. Hajian and S. Hardt, Formation and Lateral Migration of Nanodroplets via Solvent Shifting in a Microfluidic Device. Microfluid, Nanofluids, 2015, 19, 1281-1296.

27 C. J. Lan, S. Pal, Z. Li and Y. Ma, Numerical Simulations of the Digital Microfluidic Manipulation of Single Microparticles, Langmuir, 2015, 31, 9636-9645.

28 J. Koh, C. Y. Wu, H. Kittura and D. D. Carlo, Research Highlights: Microfluidically-Fabricated Materials, Lab Chip, 2015, 15, 3818-3821.

29 F. Heslot, N. Fraysse and A. Cazabat, Molecular Layering in the Spreading of Wetting Liquid Drops, Nature, 1989, 338, 640-642.

30 D. Bonn, J. Eggers, J. Indekeu, J. Meunier and E. Rolley, Wetting and Spreading, Rev. Mod. Phys., 2009, 81, 739-805.

31 T. L. Sun, L. Feng, X. F. Gao and J. Lei, Bioinspired Surfaces with Special Wettability, Acc. Chem. Res., 2005, 38, 644-652.

32 X. C. Xuan, B. Xu, D. Sintonb and D. Q. Li, Electroosmotic Flow with Joule Heating Effects, Lab Chip, 2004, 4, 230-236.

33 G. Y. Tang, C. Yang, C. J. Chai and H. Q. Gong, Modeling of Electroosmotic Flow and Capillary Electrophoresis with the Joule Heating Effect: The Nernst-Planck Equation versus the Boltzmann Distribution, Langmuir, 2003, 19, 1097510984.

34 Z. Chen, L. Feng, C. Zhang, H. Bie, G. Lei and F. L. Bai, The Light-emitting Device Consisting of Organic White-light Components, Curr. Opin. Colloid Interface Sci., 2007, 11, 28-32.

35 S. Ahrar, M. Hwang, P. N. Duncana and E. E. Hui, Microfluidic Serial Dilution Ladder, Analyst, 2014, 139, 187-190.

36 J. H. Jou, S. Kumar, M. Singh, Y. H. Chen, C. C. Chen and M. T. Lee, Carrier Modulation Layer-enhanced Organic Light-emitting Diodes, Molecules, 2015, 20, 13005-13030. 